\title{
FUNGI DETERMINED IN ANKARA UNIVERSITY TANDOĞAN CAMPUS AREA (ANKARA-TURKEY)
}

\author{
Ilgaz AKATA ${ }^{1 *}$, Deniz ALTUNTAŞ ${ }^{1}$, Şanlı KABAKTEPE ${ }^{2}$ \\ ${ }^{1}$ Ankara University, Faculty of Science, Department of Biology, Ankara, TURKEY \\ ${ }^{2}$ Turgut Ozal University, Battalgazi Vocational School, Battalgazi, Malatya, TURKEY \\ *Corresponding author: ORCID ID: orcid.org/0000-0002-1731-1302, e-mail: akata@ science.ankara.edu.tr
}

Cite this article as:

Akata I., Altuntaş D., Kabaktepe Ş. 2019. Fungi Determined in Ankara University Tandoğan Campus Area (Ankara-Turkey). Trakya Univ J Nat Sci, 20(1): 47-55, DOI: 10.23902/trkjnat.521256

Received: 02 February 2019, Accepted: 14 March 2019, Online First: 15 March 2019, Published: 15 April 2019

\begin{abstract}
The current study is based on fungi and infected host plant samples collected from Ankara University Tandoğan Campus (Ankara) between 2017 and 2019. As a result of the field and laboratory studies, 148 fungal species were identified. With the addition of formerly recorded 14 species in the study area, a total of 162 species belonging to 87 genera, 49 families, and 17 orders were listed.
\end{abstract}

Key words: Ascomycota, Basidiomycota, Ankara, Turkey.

Özet: Bu çalışma, Ankara Üniversitesi Tandoğan Kampüsü'nden (Ankara) 2017 ve 2019 yılları arasında toplanan mantar ve enfekte olmuş konukçu bitki örneklerine dayanmaktadır. Arazi ve laboratuvar çalışmaları sonucunda 148 mantar türü tespit edilmiștir. Daha önce bildirilen 14 tür dahil olmak üzere 17 ordo, 49 familya, 87 cinse mensup 162 tür listelenmiștir.

\section{Introduction}

Ankara, the capital city of Turkey, is situated in the center of Anatolia, surrounded by Çankırı in the north, Bolu in the northwest, Kırşehir, and Kırıkkale in the east, Eskisehir in the west, Konya and Aksaray in the south. Ankara University Tandoğan Campus is located in Tandoğan locality within the boundaries of Çankaya district and it is situated at an elevation between 850 and 870 meters above the sea level. The campus covers a total surface area of $195.000 \mathrm{~m}^{2}$ and includes the faculty of science, the faculty of pharmacy, the biotechnology institute, the sports complex, the rectorate, and various administrative buildings (Fig. 1). Since its first establishment, local and exotic trees, shrubs and herbaceous plants have been planted in various areas of the campus, and the current flora comprises over 300 plant species, of which approximately 60 are in tree forms.

Fungi significant members of the ecosystem by playing important roles in not only in the ecosystem itself but also in various fields including, pharmacology, food industry, and biodegradation. More than 120.000 fungal species have been described and reported to currently exist throughout the world but global fungal biodiversity has been estimated to comprise approximately 1.5 million species (Servi et al. 2010).

Studies on Turkish fungal diversity is based on a study period of more than 100 years. Over the last decade, the number of studies has significantly increased and the compiled literature data were published as checklists in different times (Bahçecioğlu \& Kabaktepe 2012, Doğan et al. 2005, Sesli \& Denchev 2008, Kabaktepe et al. 2015). Recently, data of the new studies on the Turkish macrofungal diversity were also incorporated into these data that are not included in these checklists (Acar \& Uzun 2017, Akata 2017, Akata \& Uzun 2017, Akata et al. 2018, Akgül et al. 2015, Allı et al. 2017, Altuntaş et al. 2017, Demirel et al. 2017, Doğan et al. 2018, Ekici et al. 2012, Erdoğdu \& Hüseyin 2008, Hüseyin \& Selçuk 2016, Hüseyin et al. 2016, Ișik \& Türkekul 2018, Kabaktepe et al. 2016, 2017, Kabaktepe \& Akata 2018, Öztürk et al. 2017; Selçuk \& Ekici 2014, Sesli \& Vizzini 2017, Sesli \& Liimatainen 2018, Şen et al. 2018, Uzun et al. 2014). Previous mycological investigations in the research area were conducted by Akata (2010), Akata \& Heluta (2015), Akata et al. (2009) and totally 35 fungal species reported. However, up until now, no detailed mycological research devoted to Ankara University Tandoğan Campus has been conducted.

\section{Materials and Methods}

The study was conducted between the years 2017 and 2019 in Ankara University Tandoğan Campus. Fungal specimens and the infected host plants in the campus area were collected and were brought to the laboratory for identifications. Macroscopic and some ecological features of the samples were noted in the field and the specimens 
were photographed in their natural environment. Microfungal samples were isolated from the plant material either by scraping or by thin sectioning using a razor blade, spore prints were obtained from macrofungal samples and microstructureswere examined with light microscopy. The reagents such as distilled water, Melzer's reagent, Lactophenol, $5 \% \mathrm{KOH}, \mathrm{H}_{2} \mathrm{SO}_{4}$, Cotton blue, Congo red etc. were used. Identification of the samples was performed according to Breitenbach \& Kränzlin (1984, 1986, 1991, 1995, 2000), Braun \& Cook (2012), Ellis \& Ellis (1987), Hansen \& Knudsen (1992, 1997, 2000), Jordan (2004), Kränzlin (2005), Wilson \& Henderson (1966) and Vánky (2012). The identified fungal samples are kept at Ankara University Herbarium (ANK).

\section{Results}

The taxa identified were listed in alphabetical order with notes on their habitats, host plants, collection dates, and accession numbers before which "ANK Akata \& Altuntaş" was added. Their systematics were given in accordance with the Index Fungorum (www.indexfungorum.org; accessed 25 January 2019).

\section{Division ASCOMYCOTA \\ Order Capnodiales \\ Family Cladosporiaceae}

Cladosporium herbarum (Pers.) Link: On leaf and stem of Triticum aestivum L. (Poaceae), 27.05.2018, ANK Akata \& Altuntaş 107.

Cladosporium macrocarpum Preuss: On leaf of Triticum aestivum L. (Poaceae), 17.05.2018, ANK Akata \& Altuntaş 102.

\section{Family Mycosphaerellaceae}

Mycosphaerella populnea (Sacc.) House: On leaf of Populus nigra L. (Salicaceae), 08.07.2018, ANK Akata \& Altuntaş 127; 18.08.2018, ANK Akata \& Altuntaş 156.

Stigmina carpophila (Lév.) M.B. Ellis: On leaf and fruit of Prunus domestica L. (Rosaceae), 05.06.2018, ANK Akata \& Altuntaş 118.

\section{Order Diaporthales \\ Family Gnomoniaceae}

Ophiognomonia leptostyla (Fr.) Sogonov: On leaf of Juglans regia L. (Juglandaceae), 05.07.2018, ANK Akata \& Altuntaş 123; 17.08.2018, ANK Akata \& Altuntaş 148.

\section{Order Erysiphales \\ Family Erysiphaceae}

Blumeria graminis (DC.) Speer: On leaf and stem of Avena barbata Pott ex Link (Poaceae), 28.09.2017, ANK Akata \& Altuntaş 17; On leaf and stem of T. aestivum L. (Poaceae), 14.08.2018, ANK Akata \& Altuntaş 138.

Erysiphe alphitoides (Griffon \& Maubl.) U. Braun \& S. Takam.: On leaf of Quercus robur L. (Fagaceae), 17.08.2018, ANK Akata \& Altuntaş 142; 20.09.2018, ANK Akata \& Altuntaş 173; 15.10.2018, ANK Akata \& Altuntaş 262.
Erysiphe aquilegiae DC.: On leaf and stem of Ranunculus neopolitanus Ten. (Ranunculaceae), 13.10.2018, ANK Akata \& Altuntaş 248.

Erysiphe berberidis DC.: On leaf of Berberis aquifolium Pursh (Berberidaceae), 17.08.2018, ANK Akata \& Altuntaş 145.

Erysiphe convolvuli DC.: On leaf of Convolvulus arvensis L. (Convolvulaceae), 16.10.2017, ANK Akata \& Altuntaş 28.

Erysiphe flexuosa (Peck) U. Braun \& S. Takam.: On leaf of Aesculus hippocastanum L. (Sapindaceae), 09.10.2018 ANK Akata \& Altuntaş 229.

Erysiphe heraclei DC.: On leaf, stem and fruit of Torilis arvensis (Huds.) Link (Apiaceae), 01.10.2018, ANK Akata \& Altuntaş 207.

Erysiphe lycopsidis R.Y. Zheng \& G.Q. Chen: On leaf and stem of Anchusa leptophylla Roem. \& Schult. (Boraginaceae), 01.10.2017, ANK Akata \& Altuntaş 24.

Erysiphe necator Schwein.: On leaf of Vitis vinifera $\mathrm{L}$. (Vitaceae), 25.09.2018, ANK Akata \& Altuntaş 196.

Erysiphe pisi DC.: On leaf and stem of Medicago sativa L. (Fabaceae), 23.10.2017, ANK Akata \& Altuntaş 41; 02.10.2018, ANK Akata \& Altuntaş 210.

Erysiphe polygoni DC.: On leaf and stem of Polygonum aviculare L. (Polygonaceae), 13.10.2018, ANK Akata \& Altuntaş 249.

Erysiphe syringae-japonicae (U. Braun) U. Braun \& S. Takam.: Akata \& Heluta (2015).

Erysiphe urticae (Wallr.) S. Blumer: On leaf and stem of Urtica dioica L. (Urticaceae), 25.09.2018, ANK Akata \& Altuntaş 197.

Golovinomyces cichoracearum (DC.) V.P. Heluta: On leaf and stem of Taraxacum officinale F.H. Wigg. (Asteraceae)17.08.2018, ANK Akata \& Altuntaş 150.

Golovinomyces depressus (Wallr.) V.P. Heluta: On leaf of Arctium minus (Hill) Bernh. (Asteraceae), 21.09.2018, ANK Akata \& Altuntaş 183.

Golovinomyces sordidus (L. Junell) V.P. Heluta: On leaf and stem of Plantago major L. (Plantaginaceae), 17.08.2018, ANK Akata \& Altuntaş 143.

Leveillula lactucarum Durrieu \& Rostam: On leaf of Lactuca serriola L. (Asteraceae), 09.10.2018 ANK Akata \& Altuntaş 230 .

Leveillula lappae (Castagne) U. Braun: On leaf and stem of Cirsium arvense (L.) Scop. (Asteraceae), 21.09.2018, ANK Akata \& Altuntaş 182.

Leveillula papilionacearum (Kom.) U. Braun: On leaf and stem of Medicago sativa L. (Fabaceae), 23.10.2017, ANK Akata \& Altuntaş 39; On leaf and stem of Vicia sativa L. ssp. nigra (L.) Ehrh. (Fabaceae), 06.10.2018, ANK Akata \& Altuntaş 222. 
Neoerysiphe galeopsidis (DC.) U. Braun: On leaf and stem of Lamium purpureum L. (Lamiaceae), 28.09.2018, ANK Akata \& Altuntaş 201.

Podosphaera clandestina (Wallr.) Lév.: On leaf of Cydonia oblonga Mill. (Rosaceae), 21.09.2018, ANK Akata \& Altuntaş 180.

Podosphaera dipsacacearum (Tul. \& C. Tul.) U. Braun \& S. Takam.: On leaf and stem of Scabiosa rotata M. Bieb. (Dipsacaceae), 02.10.2018, ANK Akata \& Altuntaş 212.

Podosphaera ferruginea (Schltdl.) U. Braun \& S. Takam.: On leaf and stem of Sanguisorba minor Scop. (Rosaceae), 25.09.2018, ANK Akata \& Altuntaş 194.

Podosphaera fusca (Fr.) U. Braun \& Shishkoff: On leaf and stem of Xanthium spinosum L. (Asteraceae), 17.08.2018, ANK Akata \& Altuntaş 149.

Podosphaera pannosa (Wallr.) de Bary: On leaf of Rosa canina L. (Rosaceae), 19.08.2018, ANK Akata \& Altuntaş 162.

Podosphaera plantaginis (Castagne) U. Braun \& S. Takam.: On leaf and stem of Plantago major L. (Plantaginaceae), 13.09.2017, ANK Akata \& Altuntaş 14.

Phyllactinia fraxini (DC.) Fuss: On leaf of Fraxinus angustifolia Vahl (Oleaceae), 02.10.2018, ANK Akata \& Altuntaş 215.

Phyllactinia guttata (Wallr.) Lév. On leaf of Corylus avellana L. (Betulaceae), 17.08.2018, ANK Akata \& Altuntaş 147.

Phyllactinia mali (Duby) U. Braun: On leaf and stem of Crataegus monogyna Jacq. (Rosaceae), 17.08.2018, ANK Akata \& Altuntaş 144.

Sawadaea bicornis (Wallr.) Homma: On leaf of Acer platanoides L (Aceraceae), 06.10.2018 ANK Akata \& Altuntaş 218.

\section{Order Glomerellales \\ Family Glomerellaceae}

Colletotrichum gloeosporioides (Penz.) Penz. \& Sacc.: On leaf of Pyrus communis L. (Rosaceae), 14.05.2018, ANK Akata \& Altuntaş 98; 27.05.2018, ANK Akata \& Altuntaş 109.

\section{Family Plectosphaerellaceae}

Verticillium albo-atrum Reinke \& Berthold: On leaf and stem of Morus alba L. (Moraceae), 27.05.2018, ANK Akata \& Altuntaş 108; 17.08.2018, ANK Akata \& Altuntaş 146; 25.09.2018, ANK Akata \& Altuntaş 195.

\section{Order Hypocreales}

\section{Family Hypocreaceae}

Hypomyces chrysospermus Tul. \& C. Tul.: On Xerocomellus chrysenteron (Bull.) Šutara (Boletaceae), 14.08.2018, ANK Akata \& Altuntaş 141.

\section{Order Pezizales}

Family Helvellaceae

Helvella acetabulum (L.) Quél.; On path, 05.05.2018, ANK Akata \& Altuntaş 79.

Helvella costifera Nannf.: Under Picea pungens Engelm. (Pinaceae), 17.10.2018, ANK Akata \& Altuntaş 275.

Helvella leucomelaena (Pers.) Nannf.: Under Pinus nigra J.F Arnold (Pinaceae), 14.05.2018, ANK Akata \& Altuntaş 96.

\section{Family Pyronemataceae}

Geopora arenicola (Lév.) Kers: Under Pinus nigra J.F Arnold (Pinaceae), 14.10.2018, ANK Akata \& Altuntaş 254.

Geopora sumneriana (Cooke) M. Torre: Under Cedrus libani A. Rich. (Pinaceae), 10.05.2018, ANK Akata \& Altuntaş 92.

Pulvinula convexella (P. Karst.) Pfister: Under Crataegus monogyna Jacq. (Rosaceae), 12.10.2018, ANK Akata \& Altuntaş 236.

\section{Order Pleosporales \\ Family Venturiaceae}

Venturia pyrina Aderh.: On leaf and fruit of Pyrus communis L. (Rosaceae), 18.08.2018, ANK Akata \& Altuntaş 158.

\section{Order Phyllachorales}

Family Phyllachoraceae

Polystigma rubrum (Pers.) DC.: On leaf of Prunus domestica L. (Rosaceae), 23.09.2018, ANK Akata \& Altuntaş 188; 20.09.2018, ANK Akata \& Altuntaş 171.

\section{Order Rhytismatales \\ Family Rhytismataceae}

Rhytisma acerinum (Pers.) Fr.: On leaf of Acer platanoides L. (Aceraceae), 27.09.2018, ANK Akata \& Altuntaş 200.

\section{Division BASIDIOMYCOTA \\ Order Agaricales \\ Family Agaricaceae}

Agaricus campestris L.: In meadow, 16.10.2018, ANK Akata \& Altuntaş 270; 29.10.2018, ANK Akata \& Altuntaş 575; Akata et al. (2009).

Agaricus xanthodermus Genev. In meadow, 05.06.2018, ANK Akata \& Altuntaş 112; 29.09.2018, ANK Akata \& Altuntaş 205; 14.08.2018, ANK Akata \& Altuntaş 139.

Coprinus comatus (O.F. Müll.) Pers.: In meadow, 20.09.2018, ANK Akata \& Altuntaş 174; 16.10.2018, ANK Akata \& Altuntaş 271; 26.10.2018, ANK Akata \& Altuntaş 565; Akata et al. (2009).

Crucibulum laeve (Huds.) Kambly: On dead branch of Quercus robur L. (Fagaceae), 29.09.2017, ANK Akata \& Altuntaş 19. 
Lepiota brunneoincarnata Chodat \& C. Martín: Under Aesculus hippocastanum L. (Sapindaceae), 13.10.2018, ANK Akata \& Altuntaş 247.

Lepiota cristata (Bolton) P. Kumm.: In meadow, 15.10.2018, ANK Akata \& Altuntaş 260; 17.10.2018, ANK Akata \& Altuntaş 276; Akata et al. (2009).

Lepiota lilacea Bres.: In meadow, 08.09.2017, ANK Akata \& Altuntaş 03.

Leucoagaricus leucothites (Vittad.) Wasser: In meadow, 16.10.2018, ANK Akata \& Altuntaş 269; Akata et al. (2009).

\section{Family Bolbitiaceae}

Conocybe apala (Fr.) Arnolds: Akata et al. (2009).

Conocybe tenera (Schaeff.) Fayod: In meadow, 07.10.2018 ANK Akata \& Altuntaş 223; Akata et al. (2009).

\section{Family Clavariaceae}

Clavaria fragilis Holmsk.: In meadow, 14.10.2018, ANK Akata \& Altuntaş 252.

\section{Family Cortinariaceae}

Cortinarius decipiens (Pers.) Fr.: Under Betula pendula Roth (Betulaceae), 15.09.2017, ANK Akata \& Altuntaş 16.

\section{Family Cyphellaceae}

Chondrostereum purpureum (Pers.) Pouzar: On dead branch of Quercus robur L. (Fagaceae), 13.01.2019, ANK Akata \& Altuntaş 605; Akata et al. (2009).

\section{Family Entolomataceae}

Entoloma sericeoides (J.E. Lange) Noordel.: In meadow, 28.04. 2018, ANK Akata \& Altuntaş 60.

\section{Family Hymenogastraceae}

Hebeloma crustuliniforme (Bull.) Quél.: Under Betula pendula Roth (Betulaceae), 18.10.2018, ANK Akata \& Altuntaş 279.

Hebeloma hiemale Bres.: Under Quercus robur L. (Fagaceae), 14.10.2018, ANK Akata \& Altuntaş 255.

Hebeloma mesophaeum (Pers.) Quél.: Under Picea pungens Engelm. (Pinaceae), 17.10.2018, ANK Akata \& Altuntaş 272; 10.12.2018, ANK Akata \& Altuntaş 602.

Hebeloma sinapizans (Paulet) Gillet: Under Quercus robur L. (Fagaceae), 12.10.2018, ANK Akata \& Altuntaş 237.

\section{Family Inocybaceae}

Crepidotus caspari Velen.: On stump of Cercis siliquastrum L. (Fabaceae), 13.10.2018, ANK Akata \& Altuntaş 246.

Inocybe geophylla (Bull.) P. Kumm.: Akata et al. (2009).

Inocybe grammata Quél.: Under Picea pungens Engelm. (Pinaceae), 14.10.2018, ANK Akata \& Altuntaş 251.
Inocybe griseolilacina J.E. Lange: Under Pinus nigra J.F Arnold (Pinaceae), 14.10.2018, ANK Akata \& Altuntaş 257.

Inocybe queletii Konrad: Akata et al. (2009).

Inocybe pseudohiulca Kühner: Under Picea pungens Engelm. (Pinaceae), 17.10.2018, ANK Akata \& Altuntaş 273.

Inocybe rimosa (Bull.) P. Kumm.: Under Pinus nigra J.F Arnold (Pinaceae), 05.06.2018, ANK Akata \& Altuntaş 121.

Inocybe squamata J.E. Lange: Under Populus nigra L. (Salicaceae), 14.10.2018, ANK Akata \& Altuntaş 253.

Inocybe splendens R. Heim: Under Quercus robur L. (Fagaceae), 12.10.2018, ANK Akata \& Altuntaş 235.

\section{Family Lyophyllaceae}

Calocybe ionides (Bull.) Donk: Under Pyrus communis L. (Rosaceae), 15.10.2018, ANK Akata \& Altuntaş 261.

Lyophyllum decastes (Fr.) Singer: In meadow, 02.11.2018, ANK Akata \& Altuntaş 586.

\section{Family Omphalotaceae}

Gymnopus hariolorum (Bull.) Antonín, Halling \& Noordel.: Under Pyrus communis L. (Rosaceae), 25.10.2018, ANK Akata \& Altuntaş 553.

\section{Family Physalacriaceae}

Armillaria mellea (Vahl) P. Kumm.: On stump of Quercus robur L. (Fagaceae), 28.09.2018, ANK Akata \& Altuntaş 202; Akata et al. (2009).

Flammulina velutipes (Curtis) Singer: On stump of Populus nigra L. (Salicaceae), 26.11.2017, ANK Akata \& Altuntaş 49; 15.01.2019, ANK Akata \& Altuntaş 610; Akata et al. (2009).

Strobilurus tenacellus (Pers.) Singer: On cone of Pinus nigra J.F Arnold (Pinaceae), 15.04.2018, ANK Akata \& Altuntaş 58; Akata et al. (2009).

\section{Family Pleurotaceae}

Pleurotus ostreatus (Jacq.) P. Kumm.: On Populus nigra L. (Salicaceae), 21.09.2018, ANK Akata \& Altuntaş 184; Akata et al. (2009).

\section{Family Pluteaceae}

Pluteus podospileus Sacc. \& Cub.: On stump of Pyrus communis L. (Rosaceae), 27.10.2018, ANK Akata \& Altuntaş 568.

\section{Family Psathyrellaceae}

Coprinellus disseminatus (Pers.) J.E. Lange: On stump of Populus nigra L. (Salicaceae), 07.10.2017, on stump of Quercus robur L. (Fagaceae), 17.10.2018, ANK Akata \& Altuntaş 279; Akata et al. (2009).

Coprinellus micaceus (Bull.) Vilgalys, Hopple \& Jacq. Johnson: On stump of Quercus robur L. 
(Fagaceae), 07.10.2018 ANK Akata \& Altuntaş 224; Akata et al. (2009).

Coprinopsis atramentaria (Bull.) Redhead, Vilgalys \& Moncalvo: In meadow, 06.10.2018 ANK Akata \& Altuntaş 217; 17.10.2018, ANK Akata \& Altuntaş 278; Akata et al. (2009).

Lacrymaria lacrymabunda (Bull.) Pat.: In meadow, 25.09.2018, ANK Akata \& Altuntaş 193.

Panaeolina foenisecii (Pers.) Maire: Akata et al. (2009).

Panaeolus fimicola (Pers.) Gillet: In meadow, 26.10.2018, ANK Akata \& Altuntaş 561.

Panaeolus olivaceus F.H. Møller: In meadow, 25.10.2018, ANK Akata \& Altuntaş 559.

Panaeolus papilionaceus (Bull.) Quél.: In meadow, on manure, 27.10.2018, ANK Akata \& Altuntaş 567.

Parasola conopilea (Fr.) Örstadius \& E. Larss.: In meadow, 02.10.2018, ANK Akata \& Altuntaş 214.

Parasola plicatilis (Curtis) Redhead, Vilgalys \& Hopple: In meadow, 12.10.2018, ANK Akata \& Altuntaş 240.

Psathyrella candolleana (Fr.) Maire: In meadow, 20.09.2018, ANK Akata \& Altuntaş 170; 14.10.2018, ANK Akata \& Altuntaş 250.

\section{Family Schizophyllaceae} (2010)

Schizophyllum amplum (Lév.) Nakasone: Akata

Schizophyllum commune Fr.: On dead branch of Quercus robur L. (Fagaceae), 19.10.2017, ANK Akata \& Altuntaş 30; 15.10.2018, ANK Akata \& Altuntaş 265; Akata et al. (2009).

Family Strophariaceae

Cyclocybe cylindracea (DC.) Vizzini \& Angelini: On stump of Populus nigra L. (Salicaceae), 21.09.2018, ANK Akata \& Altuntaş 185; Akata et al. (2009).

Pholiota aurivella (Batsch) P. Kumm.: Akata et al. (2009).

Pholiota lubrica (Pers.) Singer: Under Pyrus communis L. (Rosaceae), 15.10.2018, ANK Akata \& Altuntaş 263.

Pholiota populnea (Pers.) Kuyper \& Tjall.-Beuk.: Akata et al. (2009).

\section{Family Tricholomataceae}

Lepista nuda (Bull.) Cooke: In meadow, 03.10.2017, ANK Akata \& Altuntaş 25; Akata et al. (2009).

Leucocybe candicans (Pers.) Vizzini, P. Alvarado, G. Moreno \& Consiglio: Under Syringa vulgaris L. (Oleaceae), 15.10.2018, ANK Akata \& Altuntaş 259.
Melanoleuca exscissa (Fr.) Singer: In meadow, 15.10.2018, ANK Akata \& Altuntaş 260.

Tricholoma scalpturatum (Fr.) Quél.: Under Pinus nigra J.F Arnold (Pinaceae), 25.10.2018, ANK Akata \& Altuntaş 552; 10.12.2018, ANK Akata \& Altuntaş 601; 15.01.2019, ANK Akata \& Altuntaş 609.

Tricholoma terreum (Schaeff.) P. Kumm.: Akata et al. (2009).

\section{Family Tubariaceae}

Tubaria conspersa (Pers.) Fayod: In meadow, among grasses, 16.10.2018, ANK Akata \& Altuntaş 268.

\section{Order Boletales}

Family Boletaceae

Rheubarbariboletus armeniacus (Quél.) Vizzini, Simonini \& Gelardi: Akata et al. (2009).

Xerocomellus chrysenteron (Bull.) Šutara: Under Pinus nigra J.F Arnold (Pinaceae), 14.08.2018, ANK Akata \& Altuntaş 140.

\section{Family Paxillaceae}

Paxillus involutus (Batsch) Fr.: Under Picea pungens Engelm. (Pinaceae), 28.09.2017, ANK Akata \& Altuntaş 18.

\section{Family Sclerodermataceae}

Pisolithus arhizus (Scop.) Rauschert: Under Pinus nigra J.F Arnold (Pinaceae), 14.08.2018, ANK Akata \& Altuntaş 137.

Scleroderma citrinum Pers.: On path, 14.10.2018, ANK Akata \& Altuntaş 256; Akata et al. (2009).

Scleroderma verrucosum (Bull.) Pers.: Under Tilia tomentosa Moench (Malvaceae), 08.09.2017, ANK Akata \& Altuntaş 10; 23.09.2018, ANK Akata \& Altuntaş 189; 09.10.2018 ANK Akata \& Altuntaş 231; 15.10.2018, ANK Akata \& Altuntaş 264.

\section{Family Suillaceae}

Suillus collinitus (Fr.) Kuntze: Under Pinus nigra J.F Arnold (Pinaceae), 01.10.2018, ANK Akata \& Altuntaş 206; 06.10.2018, ANK Akata \& Altuntaş 274.

\section{Order Hymenochaetales \\ Family Hymenochaetaceae}

Fomitiporia punctata (P. Karst.) Murrill: On Pyracantha coccinea M.Roem. (Rosaceae) 15.10.2018, ANK Akata \& Altuntaş 258.

Fuscoporia torulosa (Pers.) T. Wagner \& M. Fisch.: On Crataegus monogyna Jacq. (Rosaceae), 12.10.2018, ANK Akata \& Altuntaş 239.

Inonotus hispidus (Bull.) P. Karst.: On hardwood, 01.10.2017, ANK Akata \& Altuntaş 21.

Phellinus igniarius (L.) Quél: On Salix babylonica L. (Salicaceae), 19.10.2017, ANK Akata \& Altuntaş 33. 
Order Phallales

Family Phallaceae

Phallus hadriani Vent.: On path, 21.09.2018, ANK Akata \& Altuntaş 179.

\section{Order Polyporales}

Family Ganodermataceae

Ganoderma adspersum (Schulzer) Donk: On stump of Acer platanoides L. (Aceraceae), 27.10.2018, ANK Akata \& Altuntaş 569. (2009).

Ganoderma applanatum (Pers.) Pat.: Akata et al.

Ganoderma lucidum (Curtis) P. Karst.: On Euonymus japonicus Thunb. (Celastraceae), 05.06.2018, ANK Akata \& Altuntaş 116.

\section{Family Meruliaceae}

Bjerkandera adusta (Willd.) P. Kars.: On stump of Quercus robur L. (Fagaceae), 15.01.2019, ANK Akata \& Altuntaş 611; Akata et al. (2009).

\section{Family Polyporaceae}

Cerioporus squamosus (Huds.) Quél.: On Robinia pseudoacacia L. (Fabaceae), 26.10.2018, ANK Akata \& Altuntaş 566; Akata et al. (2009).

Fomes fomentarius (L.) Fr.: On Salix babylonica L. (Salicaceae), 02.10.2018, ANK Akata \& Altuntaş 208; Akata et al. (2009).

Polyporus tuberaster (Jacq. ex Pers.) Fr.: On Prunus avium (L.) L. (Rosaceae), 08.09.2017, ANK Akata \& Altuntaş 05.

Trametes trogii Berk.: Akata et al. (2009).

Trametes versicolor (L.) Lloyd: Akata et al. (2009).

\section{Order Pucciniales}

Family Melampsoraceae

Melampsora allii-populina Kleb.: On leaf of Populus nigra L. (Salicaceae), 05.06.2018, ANK Akata \& Altuntaş 113.

Melampsora euphorbiae (Ficinus \& C. Schub.) Castagne: leaf and stem of Euphorbia peplus L. (Euphorbiaceae), 21.08.2018, ANK Akata \& Altuntaş 165.

Melampsora salicis-albae Kleb.: On leaf of Salix babylonica L. (Salicaceae), 08.09.2017, ANK Akata \& Altuntaş 08.

\section{Family Phakopsoraceae}

Cerotelium fici (Castagne) Arthur: On leaf of Ficus carica L. (Moraceae), 20.09.2018, ANK Akata \& Altuntaş 175.

\section{Family Phragmidiaceae}

Phragmidium mucronatum (Pers.) Schltdl.: Leaf of Rosa canina L. (Rosaceae), 09.07.2018, ANK Akata \&
Altuntaş 132; 18.08.2018, ANK Akata \& Altuntaş 153; 02.10.2018, ANK Akata \& Altuntaş 216.

Phragmidium potentillae (Pers.) P. Karst.: On leaf and stem of Potentilla reptans L. (Rosaceae), 29.09.2018, ANK Akata \& Altuntaş 204.

Phragmidium sanguisorbae (DC.) J. Schröt.: On leaf and stem of Sanguisorba minor L. (Rosaceae), 08.10.2018 ANK Akata \& Altuntaş 226.

Phragmidium tuberculatum Jul. Müll.: On leaf of Rosa canina L. (Rosaceae), 29.09.2017, ANK Akata \& Altuntaş 20.

Family Pucciniaceae

Cumminsiella mirabilissima (Peck) Nannf.: On leaf of Berberis aquifolium Pursh (Berberidaceae), 08.07.2018, ANK Akata \& Altuntaş 129.

Gymnosporangium clavariiforme (Wulfen) DC.: On leaf of Crataegus monogyna Jacq. (Rosaceae), 02.10.2018, ANK Akata \& Altuntaş 211.

Gymnosporangium confusum Plowr.: On leaf of Cotoneaster nummularius Fisch.\& C.A. Mey. (Rosaceae), 23.07. 2018, ANK Akata \& Altuntaş 135.

Puccinia acetosae (Schumach.) Körn.: On leaf and stem of Rumex crispus L. (Polygonaceae), 05.07.2018, ANK Akata \& Altuntaş 125.

Puccinia calcitrapae DC.: On leaf and stem of Taraxacum officinale F.H.Wigg. (Asteraceae), 05.06.2018, ANK Akata \& Altuntaş 119.

Puccinia crepidicola Syd. \& P. Syd.: On leaf and stem of Crepis foetida L. ssp. rhoeadifolia (M.Bieb.) Celak. (Asteraceae), 17.08.2018, ANK Akata \& Altuntaş 152.

Puccinia chondrillina Bubák \& Syd.: leaf and stem of Chondrilla juncea L. (Asteraceae), 05.06.2018, ANK Akata \& Altuntaş 114.

Puccinia cnici H. Mart.: On leaf and stem of Cirsium vulgare (Savi) Ten (Asteraceae), 02.08.2018, ANK Akata \& Altuntaş 136.

Puccinia graminis Pers.: On leaf and stem of Avena barbata Pott ex Link (Poaceae), 05.06.2018, ANK Akata \& Altuntaş 117; On leaf and stem of Triticum aestivum L. (Poaceae), 18.08.2018, ANK Akata \& Altuntaş 157.

Puccinia jasmini DC.: On leaf of Jasminum fruticans L. (Oleaceae), 05.07.2018, ANK Akata \& Altuntaş 124.

Puccinia malvacearum Bertero ex Mont.: On leaf and stem of Malva neglecta Wallr. (Malvaceae), 08.07.2018, ANK Akata \& Altuntaş 128; On leaf and stem of Alcea rosea L. (Malvaceae), 18.08.2018, ANK Akata \& Altuntaş 160.

Puccinia polygoni-amphibii Pers.: On leaf and stem of Polygonum aviculare L. (Polygonaceae), 17.08.2018, ANK Akata \& Altuntaş 151. 
Puccinia recondita Roberge ex Desm.: On leaf and stem of Hordeum bulbosum L. (Poaceae), 18.08.2018, ANK Akata \& Altuntaş 155.

Puccinia striiformis Westend.: On leaf and stem of Hordeum bulbosum L. (Poaceae), 05.06.2018, ANK Akata \& Altuntaş 122.

Uromyces fischeri-eduardii Magn.: On leaf and stem of Vicia sativa L. (Fabaceae), 19.08.2018, ANK Akata \& Altuntaş 163.

Uromyces geranii (DC.) G.H. Otth \& Wartm.: On leaf and stem of Geranium rotundifolium L. (Geraniaceae), 05.06.2018, ANK Akata \& Altuntaş 110.

Uromyces pisi-sativi (Pers.) Liro: On leaf and stem of Medicago sativa L. (Fabaceae), 18.08.2018, ANK Akata \& Altuntaş 159.

Uromyces polygoni-avicularis (Pers.) G.H. Otth: On leaf and stem of Polygonum aviculare L. (Polygonaceae), 05.06.2018, ANK Akata \& Altuntaş 115.

Uromyces rumicis (Schum.) Wint: On leaf and stem of Rumex crispus L. (Polygonaceae), 18.08.2018, ANK Akata \& Altuntaş 154.

Uromyces tinctoriicola Magnus: On leaf and stem of Euphorbia peplus L. (Euphorbiaceae), 08.07.2018, ANK Akata \& Altuntaş 131.

Uromyces trifolii (R. Hedw.) Lév.: On leaf and stem of Trifolium pratense L. (Fabaceae), 05.06.2018, ANK Akata \& Altuntaş 120.

\section{Family Uropyxidaceae}

Tranzschelia discolor (Fuckel) Tranzschel \& M.A. Litv. On leaf of Prunus domestica L. (Rosaceae), 05.07.2018, ANK Akata \& Altuntaş 126.

Tranzschelia pruni-spinosae (Pers.) Dietel: On leaf of Prunus armeniaca L. (Rosaceae), 23.07. 2018, ANK Akata \& Altuntaş 134; On Prunus domestica L. (Rosaceae), 19.08.2018, ANK Akata \& Altuntaş 161.

\section{Order Russulales}

\section{Family Amylostereaceae}

Amylostereum areolatum (Chaillet ex Fr.) Boidin: Akata et al. (2009).

\section{Family Peniophoraceae}

Gloiothele lactescens (Berk.) Hjortstam: On stump of Quercus robur L. (Fagaceae), 27.10.2018, ANK Akata \& Altuntaş 570.

\section{Family Russulaceae}

Russula pallidospora J. Blum ex Romagn.: Under Quercus robur L. (Fagaceae), 20.09.2018, ANK Akata \& Altuntaş 169.

\section{Family Stereaceae}

Stereum hirsutum (Willd.) Pers.: On stump of Quercus robur L. (Fagaceae), 23.10.2017, ANK Akata \& Altuntaş 35; 09.10.2018 ANK Akata \& Altuntaş 232; Akata et al. (2009).

\section{Order Ustilaginales \\ Family Ustilaginaceae}

Ustilago avenae (Pers.) Rostr.: On fruit of Avena barbata Pott ex Link (Poaceae) 12.09.2017, ANK Akata \& Altuntaş 12 .

\section{Discussion}

A total of 162 fungal species belonging to 87 genera within 49 families were determined in the study area. The determined fungi included 47 Ascomycota (Erysiphaceae 30, Helvellaceae and Pyronemataceae 3, Cladosporiaceae and Mycosphaerellaceae 2, Gnomoniaceae, Glomerellaceae, Plectosphaerellaceae, Hypocreaceae, Venturiaceae, Phyllachoraceae and Rhytismataceae 1) and 115 Basidiomycota (Pucciniaceae 23, Psathyrellaceae 11, Inocybaceae 9, Agaricaceae 8, Polyporaceae and Tricholomataceae 5, Hymenochataceae, Hymenogastraceae, Phragmidiaceae and Strophariaceae 4, Ganodermataceae, Melampsoraceae, Physalacriaceae and Sclerodermataceae 3, Bolbitiaceae, Boletaceae, Lyophyllaceae and Schizophyllaceae 2, Clavariaceae, Cortinariaceae, Cyphellaceae, Entolomataceae, Meruliaceae, Omphalotaceae, Pleurotaceae, Pluteaceae, Tubariaceae, Paxillaceae, Suillaceae, Phallaceae, Phakopsoraceae, Amylostereaceae, Peniophoraceae, Russulaceae, Stereaceae and Ustilaginaceae 1) species.

Eighty-five of one hundred and sixty-two species determined in the campus area were parasite infecting plant tissues except for the fungicolous species (Hypomyces chrysospermus). Plant parasitic species belongs to Erysiphales or powdery mildews (30 species), Pucciniales or rusts (31 species), Ustilaginales or smuts (1), larger basidiomycete fungi (11) and other microscopic ascomycete fungi (11). Seventy-seven species reported from the research area are saprobes, among which 63 are terricolous and the number of lignicolous species is 14 .

Although 15 species (Helvella leucomelaena, Agaricus campestris, Coprinus comatus, Leucoagaricus leucothites, Lyophyllum decastes, Armillaria mellea, Flammulina velutipes, Pleurotus ostreatus, Cyclocybe cylindracea, Lepista nuda, Melanoleuca exscissa, Tricholoma terreum, Xerocomellus chrysenteron, Suillus collinitus, and Cerioporus squamosus) are suitable for human consumption, 17 species (Agaricus xanthodermus, Inocybe geophylla, I. grammata, I. griseolilacina, I. queletii, I. pseudohiulca, I. squamata, I. rimosa, Lepiota brunneoincarnata, L. cristata, Leucocybe candicans, Paxillus involutus, Hebeloma crustuliniforme, $H$. mesophaeum, H. sinapizans, Sleroderma citrinum and $S$. verrucosum) are determined as poisonous fungi in the campus area. Among them, L.brunneoincarnata is a deadly poisonous fungus containing alpha-amanitin which is responsible for the most deadly mushroom poisoning cases in Turkey (Akata et al. 2015). No poisoning case has been reported from the campus area so 
far because no one in the campus has been interested in collecting and consuming the mushrooms found inside the campus.

One hundred and twenty-seven species were determined as new species for campus area and 5 of them (Hypomyces chrysospermus, Calocybe ionides, Gloiothele lactescens, Inocybe griseolilacina and Pulvinula convexella) were recorded for the second time in Turkey.

\section{References}

1. Acar, İ. \& Uzun Y. 2017. Türkiye Mikobiyotası İçin İlginç Bir Yarı-Serbest Morel Kaydı (Morchella populiphila M. Kuo, M.C. Carter \& J.D. Moore). Mantar Dergisi, 8(2): 125-128.

2. Akata, I. 2010. Türkiye mikobiyotası için yeni bir kayıt, Schizophyllum amplum (Lev.) Nakasone. Ot Sistematik Botanik Dergisi, 17(2): 155-163.

3. Akata, I. 2017. Macrofungal Diversity of Belgrad Forest (İstanbul). Kastamonu Üniversitesi Orman Fakültesi Dergisi, 17(1): 150-164.

4. Akata, I. \& Heluta, V.P. 2015. First record of Erysiphe syringae-japonicae in Turkey. Mycotaxon, 130: 259-264.

5. Akata, I. \& Uzun, Y. 2017. Macrofungi determined in Uzungöl Nature Park (Trabzon). Trakya University Journal of Natural Sciences, 18(1): 15-24.

6. Akata, I., Doğan, H.H., Körüklü, T. \& İslek, C. 2009. Ankara Üniversitesi Tandoğan Kampüsü Makrofunguslar1. Kafkas Üniversitesi Fen Bilimleri Enstitüsü Dergisi, 2(1): 15-19.

7. Akata I., Kaya E., Yılmaz İ., Bakırcı S. \& Bayram R. 2015. Türkiye'de Yetişen Alfa Amanitin İçeren Mantarlar. Düzce Tlp Fakültesi Dergisi, 17(1): 39-44.

8. Akata, I., Kabaktepe, Ş., Sevindik, M. \& Akgül, H. 2018. Macrofungi determined in Yuvacık Basin (Kocaeli) and its close environs. Kastamonu Üniversitesi Orman Fakültesi Dergisi, 18(2): 152-163.

9. Akgül, H., Ergül, C.C., Yılmazkaya, D., Akata, I., Selçuk, F. \& Hüseyin, E. 2015. Diversity of Microfungi on Fagaceae in Uludağ Forests. Oxidation Communications, 38(3): 1529-1538.

10. Allı, H., Candar, S.S. \& Akata, I. 2017. Macrofungal Diversity of Yalova Province. Mantar Dergisi, 8(2): 76-84.

11. Altuntaş, D., Allı, H. \& Akata, I. 2017. Macrofungi of Kazdağı National Park (Turkey) and its close environs. Biological Diversity and Conservation, 10(2): 17-25.

12. Bahçecioğlu, Z. \& Kabaktepe, Ş. 2012. Checklist of rust fungi in Turkey. Mycotaxon, 119: 494.

13. Braun, U. \& Cook, R.T.A. 2012. Taxonomic manual of the Erysiphales (powdery mildews). CBS Biodiversity Series II, CBS-KHAW Fungal Biodiversity Centre, Utrecht, The Netherlands, $707 \mathrm{pp}$.

14. Breitenbach, J. \& Kränzlin, F. 1984. Fungi of Switzerland, Vol: 1, Ascomycetes. Verlag Mykologia CH-6000 Luzern 9, Switzerland, $310 \mathrm{pp}$.

\section{Acknowledgement}

We thank Ankara University Research Fund (Project No: 18B0430001) for their financial support. Dr. İsa Başköse and Dr. Ergin Şahin are also thanked for their valuable contributions in the field.

Editor-in-Chief note: Author Ilgaz Akata is a member of Editorial Board of Trakya University Journal of Natural Sciences. However, he wasn't involved in the decision process during manuscript evaluation.

15. Breitenbach, J. \& Kränzlin, F. 1986. Fungi of Switzerland, Vol: 2, Nongilled Fungi. Verlag Mykologia CH-6000 Luzern 9, Switzerland, 412 pp.

16. Breitenbach, J. \& Kränzlin, F. 1991. Fungi of Switzerland, Vol: 3, Boletes and Agarics 1. Part. Verlag Mykologia CH6000 Luzern 9, Switzerland, 361 pp.

17. Breitenbach, J. \& Kränzlin, F. 1995. Fungi of Switzerland, Vol: 4, Agarics 2. Part. Verlag Mykologia CH-6000 Luzern 9, Switzerland, $368 \mathrm{pp}$.

18. Breitenbach, J. \& Kränzlin, F. 2000. Fungi of Switzerland, Vol: 5, Agarics 3. Part. Verlag Mykologia CH-6000 Luzern 9, Switzerland, $338 \mathrm{pp}$

19. Demirel, K., Uzun, Y., Keleş, A., Akçay, M.E. \& Acar, İ. 2017. Macrofungi of Karagöl-Sahara National Park (Şavşat-Artvin/Turkey). Biological Diversity and Conservation, 10(2): 32-40.

20. Doğan, H.H., Öztürk, C., Kaşık, G. \& Aktaş, S. 2005. A Checklist of Aphyllophorales of Turkey. Pakistan Journal of Botany. 37(2): 459-485.

21. Doğan, H.H., Bozok, F. \& Taşkın, H. 2018. A new species of Barssia (Ascomycota, Helvellaceae) from Turkey. Turkish Journal of Botany, 42: 636-643.

22. Ekici, T., Erdogdu, M., Aytaç, Z. \& Suludere, Z. 2012. Septoria species in Kibris Village Valley (Ankara, Turkey). Nova Hedwigia Band, 95(3-4): 483-491.

23. Ellis, B.M. \& Ellis, J.P. 1987. Microfungi on Land Plants. Croom Helm, London \& Sydney, 818 pp.

24. Erdoğdu, M. \& Hüseyin, E. 2008. Microfungi of Kurtboğazı Dam (Ankara) and its environment. Ot Sistematik Botanik Dergisi, 14(1): 131-150.

25. Hansen, L. \& Knudsen, H. 1992. Nordic Macromycetes, Volume 2, Polyporales, Boletales, Agaricales, Russulales. Nordsvamp, Copenhagen, Denmark, 474 pp.

26. Hansen, L. \& Knudsen, H. 1997. Nordic Macromycetes, Volume 3, Heterobasidoid, Aphyllophoroid, and Gastromycetoid Basidiomycetes. Nordsvamp, Copenhagen, Denmark, 444 pp.

27. Hansen, L. \& Knudsen, H. 2000. Nordic Macromycetes, Volume 1, Ascomycetes. Nordsvamp, Copenhagen, Denmark, 209 pp.

28. Hüseyin E. \& Selçuk, F. 2016. Pileolaria azerii (Uredinales), a new rust species from Turkey. Sydowia, 68: $1-6$. 
29. Hüseyin, E., Selçuk, F. \& Ekici K. 2016. Acrodictys, Corynespora, Karstenula, Oncopodium, and Sporocadus: new genera for Turkey. Mycotaxon, 131: 331-335.

30. Index fungorum: www.indexfungorum.org; accessed 25 January 2019.

31. Işık, H. \& Türkekul, İ. 2018. A New Record for Turkish Mycota from Tokat Province: Arachnopeziza aurelia (Pers.) Fuckel. Mantar Dergisi, 9(1): 54-57.

32. Jordan, M. 2004. The Encyclopedia of Fungi of Britain and Europe. Frances Lincoln, London, 384 pp.

33. Kabaktepe, S.., Heluta, V.P. \& Akata, I. 2015. Checklist of Powdery mildews (Erysiphales) in Turkey. Biological Diversity and Conservation, 8(3): 128-146.

34. Kabaktepe, S., Mutlu, B., Karakuş, Ş. \& Akata, I. 2016. Puccinia marrubii (Pucciniaceae), a new rust species on Marrubium globosum subsp. globosum from Niğde and Malatya in Turkey. Phytotaxa, 272(4): 277-286.

35. Kabaktepe, Ş., Akata, I., Siahaan, S.A.S., Takamatsu, S. \& Braun, U. 2017. Powdery mildew (Ascomycota, Erysiphales) on Fontanesia phillyreoides and Jasminum fruticans in Turkey. Mycoscience, 58: 30-34.

36. Kabaktepe, Ş. \& Akata, I. 2018. Septoria Sacc. (Mycosphaerellales) Species Determined in Aladağlar and Bolkar Mountains (Turkey). Mantar Dergisi, 9(2): 142147.

37. Kränzlin, F. 2005. Fungi of Switzerland, Volume 6, Russulaceae 2. Verlag Mykologia, Switzerland, $319 \mathrm{pp}$.

38. Öztürk, C., Pamukçu, D. \& Aktaş, S. 2017. Macrofungi of Nallihan (Ankara) District. Mantar Dergisi, 8(1): 60-67.
39. Selçuk, F. \& Ekici, K. 2014. A new species of Manoharachariella (Hyphomycetes) from Central Anatolia, Turkey. Mycosphere, 5 (3): 419-423.

40. Servi, H., Akata, I. \& Çetin, B. 2010. Macrofungal diversity of Bolu Abant Nature Park (Turkey). African Journal of Biotechnology, 9(24): 3622-3628.

41. Sesli, E. \& Denchev, C.M. 2008. Checklists of the myxomycetes, larger ascomycetes, and larger basidiomycetes in Turkey. Mycotaxon, 106: 65-67.

42. Sesli, E. \& Vizzini, A. 2017. Two new Rhodocybe species (sect. Rufobrunnea, Entolomataceae) from the East Black Sea coast of Turkey. Turkish Journal of Botany, 41: 200210 .

43. Sesli, E. \& Liimatainen, K. 2018. Cortinarius conicoumbonatus (Cortinarius subgen. Telamonia sect. Hinnulei): a new species from spruce-beech forests of the East Black Sea Region of Turkey. Turkish Journal of Botany, 42: 327-334.

44. Şen, İ., Allı, H. \& Çöl, B. 2018. Tricholoma bonii, A New Record for Turkish Mycota and Notes on its Taxonomic Status Based on Morphological and Molecular Evidence. Turkish Journal of Life Sciences, 3(1): 200-204.

45. Uzun, Y., Acar, İ., Akçay, M.E. \& Akata, I. 2014. Additions to the Turkish Discomycetes. Turkish Journal of Botany, 38: 617-622.

46. Vánky, K. 2012. Smut Fungi of the World. APS press, St. Paul. Minnesota, $1480 \mathrm{pp}$.

47. Wilson, L.M. \& Henderson, D.M. 1966. British Rust Fungi. Cambridge University Press, 384 pp. 